\title{
Ex-Situ Electron Microscopy Study of Solid Electrolyte Interphase Formed by Charge-Discharge Reaction of Silicon Negative Electrode in Lithium-Ion Secondary Battery $^{* 1}$
}

\author{
Yutaka Shimauchi ${ }^{1,2}$, Sachi Ikemoto ${ }^{1}$, Shigekazu Ohmori ${ }^{1}$ and Takaomi Itoi ${ }^{2, * 2}$ \\ ${ }^{1} J F E$ Techno-Research Corporation, Chiba 260-0835, Japan \\ ${ }^{2}$ Department of Mechanical Engineering, Chiba University, Chiba 263-8522, Japan
}

A SEI (Solid Electrolyte Interphase) is formed on the surface layer of the negative electrode active material of a lithium ion secondary battery (LIB) during the initial charging process, and its morphology and structure significantly affect performance and safety. In this study, by conducting ex situ experiments, SEM, TEM and STEM-EELS observations were performed on Si negative electrodes under charge state within an actual battery and Si negative electrodes directly charged on a TEM thin film, revealed morphology and structure of the SEI. All of the processes from specimen preparation for electron microscopy observation to specimen transport were performed under non-atmospheric exposure conditions.

The SEI on the surface of the Si negative electrode grew thicker as the charge depth increased. On the other hands, Li $\mathrm{x}_{\mathrm{X}} \mathrm{Si}$ amorphous phase due to the lithiation by solid-state reaction was confirmed inside the $\mathrm{Si}$ negative electrode. It was found that $\mathrm{Li}_{2} \mathrm{O}$ was formed on the most surface of the Si negative electrode at the initial stage of charging, and the SEI was mainly composed of $\mathrm{Li}_{2} \mathrm{O}$. The SEI of about $1 \mu \mathrm{m}$ was observed on the Si negative electrode after $40 \%$ charge, and the thickness of the SEI decreased to less than $1 / 5$ after discharge.

[doi:10.2320/matertrans.MT-M2021070]

(Received April 21, 2021; Accepted May 10, 2021; Published June 11, 2021)

Keywords: solid electrolyte interphase, Si negative electrode, microstructure, ex-situ TEM, non-atmospheric exposure

\section{Introduction}

Since lithium ion secondary batteries play a central role as energy storage devices, material development oriented toward high capacities is desirable. ${ }^{1)}$ To achieve this goal, attention has been focused on $\mathrm{Si}$, which is expected to realize an increase of approximately 10 times in the capacity-toweight ratio in comparison with carbon materials in negative electrodes capable of storing larger quantities of $\mathrm{Li}^{2}{ }^{2}$ In studies of electrochemical lithiation, anisotropy of the $\mathrm{Li}$ intrusion direction in $\mathrm{Si}$ crystals via the charging reaction has been reported based on first-principles calculations as well as other simulation techniques, in situ transmission electron microscopy (TEM) imaging and ex-situ observations under non-atmospheric exposure conditions. ${ }^{3-8)}$ In a lithium ion battery (LIB), a passivation layer known as solid electrolyte interphase (SEI) is formed at the interface between the negative electrode and electrolyte during the initial charging process. It is known that the morphology and structure of the SEI significantly affect the performance and safety of the battery. $\left.{ }^{9}, 10\right)$ The SEI formation mechanism and its structural properties have also been investigated via firstprinciples calculations, ${ }^{11,12}$ ) and it has been reported that the SEI consists of multiple inorganic and organic compounds, including Li oxides, fluorides, and carbonates. ${ }^{13)}$ Although SEI formation during the initial charging process initiates due to the reductive decomposition products of the electrolyte, mainly at the surface of the negative electrode active material, and proceeds via electron transfer, this reaction is complex, and the composition and structure of the SEI are thought to change depending on the type of electrode

\footnotetext{
${ }^{* 1}$ This Paper was Originally Published in Japanese in Japan Inst. Met Mater. 84 (2020) 382-390.

${ }^{* 2}$ Corresponding author, E-mail: itoi@faculty.chiba-u.jp
}

material, type of electrolyte, ambient temperature, and charging conditions. Various models have been proposed based on first-principles calculations. These include a model in which the reductive decomposition products of the electrolyte accumulate directly on the negative electrode surface and a model in which these products do not accumulate immediately on the negative electrode surface but form aggregates by reacting in the electrolyte at a slight distance from the negative electrode and then accumulate on the surface of the negative electrode. ${ }^{14)}$ Therefore, identification of the component substances of the SEI and clarification of their formation position and morphology through experimental techniques will provide crucial knowledge for modeling the SEI formation mechanism through computational science. Considering the nanometer size of the SEI in the initial charging process, TEM is the ideal tool to perform SEI observations. However, there are only few examples of direct observation and discussion of SEI formation on a Si negative electrode during charge/discharge processes using an actual battery structure. Techniques for observing the negative electrode in the charged state are limited because the charged electrode contains $\mathrm{Li}$, which is extremely active when exposed to moisture and air. For example, Nie et al. reported the results of observations of the microstructural change after charging when graphite particles were dispersed on the TEM specimen stage under nonatmospheric exposure conditions. ${ }^{15)}$ The authors constructed a simple lithium ion cell by surrounding the TEM stage with dispersed graphite with an ordinary graphite negative electrode, and they were thus able to observe the cell after charging. However, due to the difference in the electrical resistance of the TEM stage and the surrounding negative electrode as well as other possible factors, it is necessary to determine whether a uniform charging reaction can actually take place in these conditions. As this suggests, operand 
experiments aimed at examining the microstructural changes in a negative electrode during charge/discharge processes are frequently problematic due to the difficulty of handling the negative electrode in the charged state.

In this work, we fabricated an actual battery using $\mathrm{Si}$, which is a promising high capacity material for use as the negative electrode active material of lithium ion secondary batteries. Furthermore, we carried out microstructural observations of the SEI formation process caused by the charge/discharge reaction and the changes in the internal microstructure of the Si negative electrode using electron microscopy. Specimens for TEM observation were prepared via the focused ion beam (FIB) technique in order to appropriately evaluate the observation position, and the SEI after charging and discharging was compared and discussed. When attempting to observe the SEI formed on the surface layer of the negative electrode active material during charging, damage caused by the ion beam in the FIB method is a concern. Therefore, we prepared an actual battery that could be charged directly on a TEM thin film (Si negative electrode) which was thinly sliced via ion milling. TEM observations of these Si negative electrodes were then carried out after inducing a uniform charging reaction in the entire specimen. In the observation and analysis of the charge/discharge states of the battery, ex-situ experiments were conducted under strict environmental control to prevent contact with atmosphere in all processes, from disassembly of the battery to preparation and transport of the electron microscopy specimens.

\section{Experimental Methods}

\subsection{Preparation of the lithium ion secondary cell}

The negative electrodes were prepared by coating a metal foil collector $(\mathrm{Cu}$ foil) having a thickness of $20 \mu \mathrm{m}$ with $\mathrm{Si}$ single crystal powder:acetylene black:polyvinylidene fluoride $=80: 10: 10$ mass $\%$ as the negative electrode material. They were then dried and pressed. Acetylene black was used to reduce the electrical resistance of the electrode. The average particle size of the $\mathrm{Si}$ single crystal powder was $10 \mu \mathrm{m}$, and the coating density of the electrode was 2.1 $\mathrm{mg} / \mathrm{cm}^{2}$ per unit surface area. Since only the negative electrode was evaluated in this experiment, a Li metal foil was used in the counter electrode (positive electrode) to eliminate the influence of the positive electrode active material, and the secondary cell structure was created using a polyethylene separator with a thickness of $20 \mu \mathrm{m}$. Figure 1 shows the photograph of the prepared battery. The electrodes were punched to a diameter $(\varphi)$ of $16 \mathrm{~mm}$ and were used to

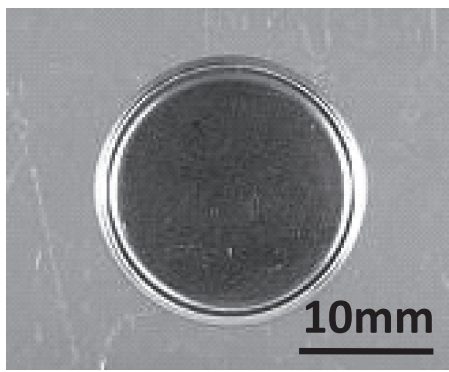

Fig. 1 Image of the coin-type battery. construct coin batteries. The electrolytic solution was a mixture of $1 \mathrm{~mol} / \mathrm{L}$ of $\mathrm{LiPF}_{6}$ in a solvent consisting of ethylene carbonate and diethyl carbonate with a volume ratio of $1: 1$.

\subsection{Charging/discharging and disassembly of the lithi- um ion secondary cell}

The insertion and desorption states of $\mathrm{Li}$ in the Si particles were observed and analyzed at various states of charge (SOCs). The SOC was adjusted through the charge/discharge device assuming $4.2 \mathrm{Ah} / \mathrm{g}$ as the theoretical capacity (upper limit in the fully charged state) of Si. A CompactStat (Ivium Technologies) electrochemical interface was used for charging/discharging. For scanning electron microscopy (SEM) observations of possible differences in the charged state, batteries with SOCs of $40 \%$ and $100 \%$ were prepared. A battery which was fully discharged $(\mathrm{SOC}=0 \%)$ after being charged to a SOC of $40 \%$ was also prepared. After charging, the coin batteries were disassembled in a glovebox in which the air had been replaced to a sufficiently low level with high purity Ar gas. The environment in the glovebox was maintained at a dewpoint of $-80^{\circ} \mathrm{C}$ or less and at an oxygen concentration of $1 \mathrm{ppm}$ or less. The coin batteries were disassembled in the glovebox immediately after charging or discharging. The samples for analysis were prepared by taking only the separated negative electrode, thoroughly cleaning the electrolyte with pure dimethyl carbonate, and drying.

\subsection{SEM observations}

After disassembly in the glovebox, the cross section of the cleaned negative electrodes was observed via FE-SEM (S-4800, Hitachi High-Tech Corporation). The specimens for cross-sectional observations were prepared using an ion milling system (IM4000, Hitachi High-Tech), and a dedicated transfer vessel was used to transfer the specimens from the glovebox to the ion milling system. This transfer vessel has an airtight structure which can maintain an inert atmosphere or vacuum around a specimen and makes it possible to transfer specimens between devices consistently without exposure to atmosphere. The same type of vessel was also used to transfer the milled specimens to the SEM setup without exposure to atmosphere. Since the reaction of the charged negative electrode with air is particularly remarkable and degradation of the specimens with time is a concern, the sequence of processes from disassembly to SEM observations was performed quickly and continuously. An accelerating voltage of $4.0 \mathrm{kV}$ and a beam current of $0.2 \mathrm{~mA}$ were used for ion milling.

\subsection{TEM and STEM observations and EELS analysis}

The cross sections of the $\mathrm{Si}$ particles in the negative electrodes charged to a SOC of $40 \%$ and discharged from a SOC of $40 \%$, which were observed via SEM in section 2.3, were also observed via TEM and STEM (ARM200F, JEOL) at an accelerated voltage of $200 \mathrm{kV}$. Furthermore, EELS (Enfinium, GATAN, Inc.) analysis was conducted. The specimens for TEM observations were prepared in the same manner as those for SEM observations; that is, the cells were disassembled in a glovebox, and the cleaned and dried 
negative electrodes were transferred to the FIB (VERSA 3D, FEI) using an FIB transfer vessel. Regarding the thin film preparation conditions for the FIB, the accelerating voltage and beam current were set to $30 \mathrm{kV}$ and $30 \mathrm{nA}$, respectively, up to a film thickness of $500 \mathrm{~nm}$. The beam diameter was narrowed by reducing the beam current in stages, and, for a thickness below $200 \mathrm{~nm}$, the FIB process was carried out with the accelerating voltage and beam current reduced to $10 \mathrm{kV}$ and several pA, respectively. Since it was not possible to transfer the specimens directly from the FIB device to the TEM while retaining non-atmospheric exposure conditions, the specimens from the FIB system were returned once to the glovebox, where they were then transferred to a different non-atmospheric exposure transfer vessel.

\subsection{Preparation of dedicated negative electrodes for TEM observations, charging, and TEM observa- tions}

Considering the possibility of degradation of the SEI caused by FIB during the preparation of the thin film specimens for TEM observations, the Si negative electrodes were charged after the thin film preparation, and the SEI was imaged. Here, a proportion of the electrode film (Si negative electrode film) prepared as described in section 2.1 was punched to a diameter of $3 \mathrm{~mm}$, and the thin films were prepared using an Ar ion milling device (Dual Ion Mill 600, GATAN) under liquid nitrogen cooling. An incident angle of $12^{\circ}$, a specimen rotation speed of $5 \mathrm{rpm}$, an accelerating voltage of $4.0 \mathrm{kV}$, and a beam current of $0.4 \mathrm{~mA}$ were used for Ar ion milling. Firstly, the Si negative electrode thin films were observed via TEM before charging. The thin film specimens were then charged, and TEM was used to image the same location as before charging in order to investigate the SEI formed after charging. Figure 2(a) shows an image of a HS (Hohsen) flat cell, and Fig. 2(b) shows a schematic diagram of the structure inside the HS flat cell used for charging. As for the experiment described in section 2.1, a $\mathrm{Li}$ metal foil was used in the counter electrode (positive electrode), and a half-cell structure using a polypropylene separator with a thickness of $20 \mu \mathrm{m}$ was constructed as the cell structure. The electrolytic solution used here was prepared by mixing $1 \mathrm{~mol} / \mathrm{L}$ of $\mathrm{LiPF}_{6}$ in a solvent consisting of ethylene carbonate and diethyl carbonate with a volume ratio of $1: 1$, and the cell was charged using the abovementioned CompactStat (Ivium Technologies). The thin film prepared for TEM observations was weighed, and the SOC with respect to the theoretical capacity was calculated based on the blending ratio of the electrode film. TEM observations were conducted after charging to a SOC of $20 \%$. In this experiment, charging was limited to a SOC of $20 \%$ because of the concern that it would not be possible to maintain the thin film state due to the swelling of the Si particles caused by charging. After disassembling the battery, the specimen was transferred to the TEM setup via the glovebox so as to prevent exposure to atmosphere, in the same manner as described in section 2.4.

\section{Results and Discussion}

\subsection{SEM observations after charging and discharging}

Figure 3 shows the charging and discharging curves of a cell (negative electrode) which was charged to SOC $=40 \%$

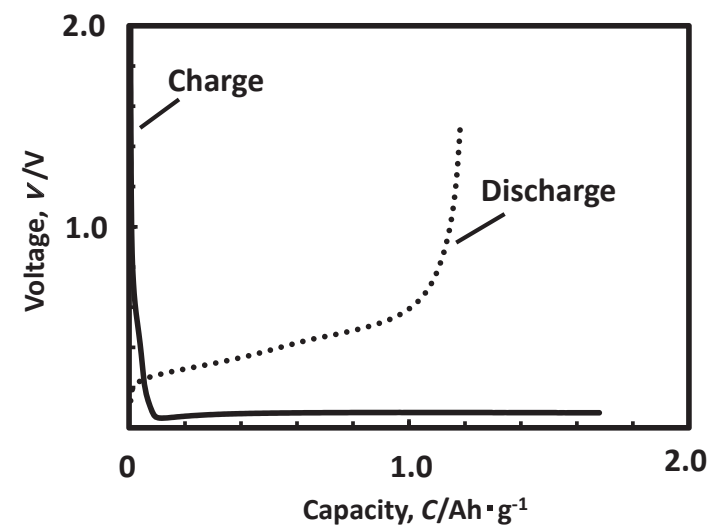

Fig. 3 Charging and discharging curves of an Si negative electrode. (a)

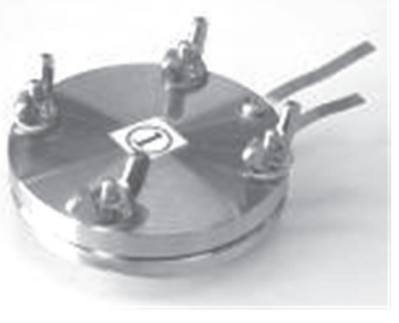

(b)

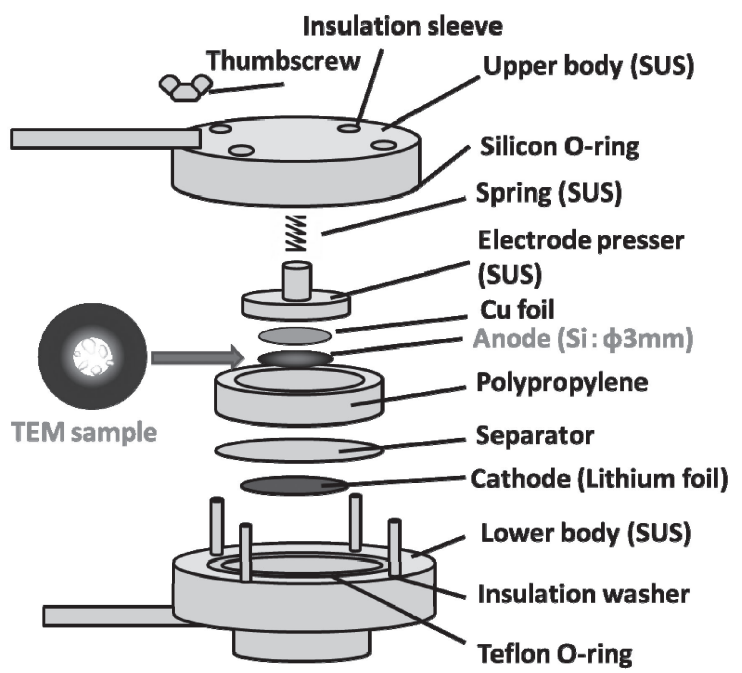

Fig. 2 (a) Image of a HS flat cell. (b) Schematic diagram of the structure inside the HS flat cell used for charging. 


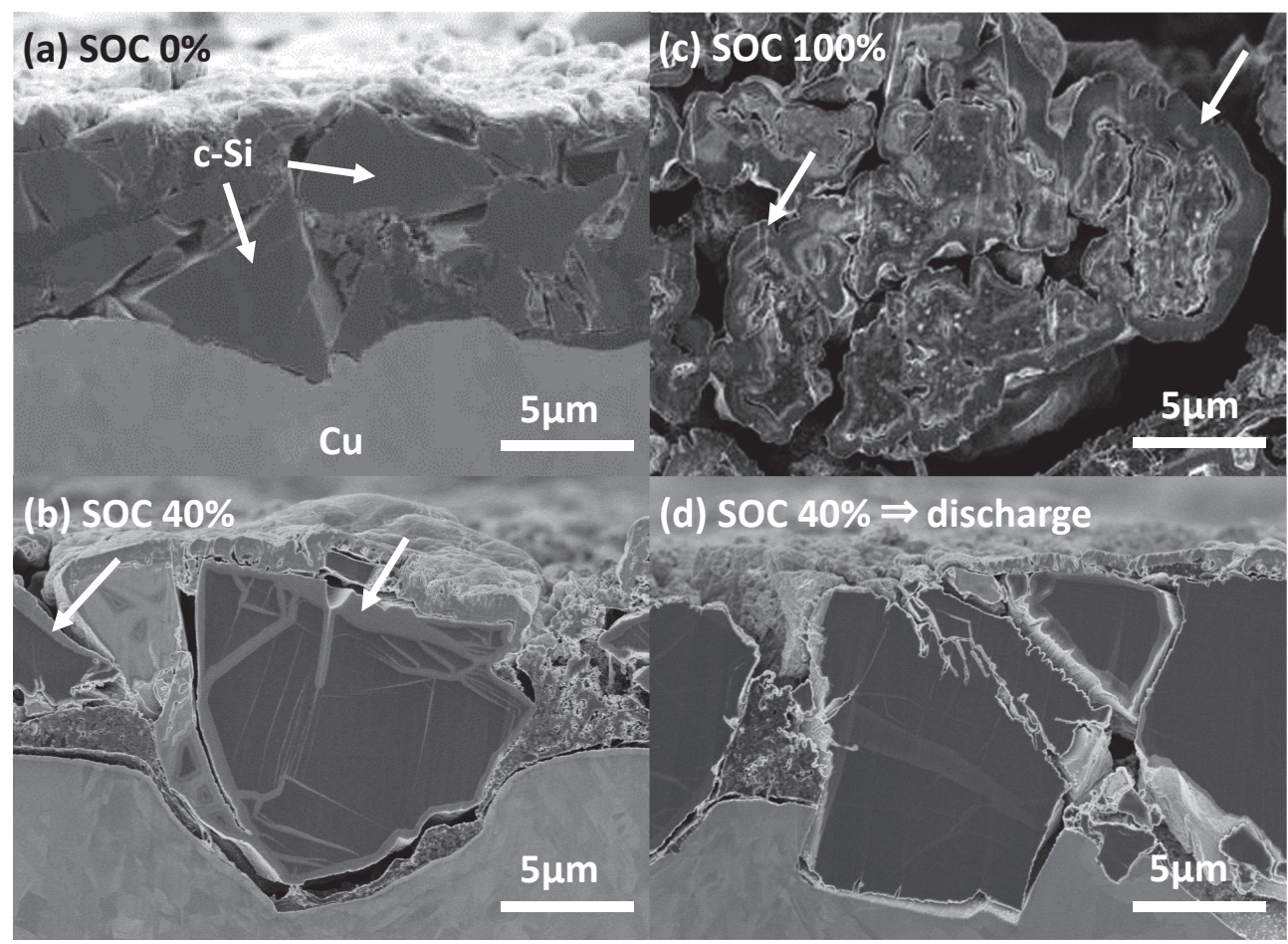

Fig. 4 Cross-sectional SEM images of the Si negative electrodes with different SOCs. (a) $0 \%$ (before charging), (b) $40 \%$, (c) $100 \%$ (fully charged), and (d) discharged (SOC $40 \%$ to $0 \%$ ) states.

and then discharged. Since the charging and discharging rates were set to a $\mathrm{C}$-rate equivalent to $0.1 \mathrm{C}$, the charging and discharging times for SOC $=40 \%$ were approximately $4 \mathrm{~h}$. From this figure, it can be seen that the voltage decreased due to charging from around $2.8 \mathrm{~V}$, which was the open circuit voltage (OVC) before charging. Furthermore, upon discharging after charging to $\mathrm{SOC}=40 \%$, which is equivalent to approximately $1.7 \mathrm{Ah} / \mathrm{g}$, only about $1.2 \mathrm{Ah} / \mathrm{g}$ was discharged. It is believed that this occurs due to the fact that the $\mathrm{Li}$ ions transferred into the Si negative electrode remain in the electrode, resulting in an irreversible loss of capacity.

Figure 4 shows cross-sectional SEM images of the $\mathrm{Si}$ negative electrodes (a) before charging (SOC $=0 \%)$, (b) after charging ( $\mathrm{SOC}=40 \%)$, (c) when fully charged (SOC $=$ $100 \%$ ), and (d) after being discharged from SOC $=40 \%$. In Fig. 4(a), which shows the cross section in the initial state before charging, the $\mathrm{Si}$ particles (c-Si) in the negative electrode are pressed into the $\mathrm{Cu}$ foil by the pressing device in order to increase the density and reduce the electrical resistance of the negative electrode. In Fig. 4(b), after charging to $\mathrm{SOC}=40 \%$, the SEI corresponds to the area with gray contrast indicated by the arrows. A SEI with a thickness from about 0.5 to $1.3 \mu \mathrm{m}$ exists uniformly around the $\mathrm{Si}$ particles, and areas of linear contrast (traces of $\mathrm{Li}$ intrusion) can be observed in the Si particles. The regularity of $\mathrm{Li}$ intrusions into the $\mathrm{Si}$ single crystals during charging was confirmed, and it is attributed to the crystal orientation dependency of lithiation. Comparing Figs. 4(b) and 4(c), it can be seen that the SEI has grown to a considerable thickness, as indicated by the dark contrast shown by the arrows. As a result, the $\mathrm{Si}$ particles were unable to maintain their original shape and were destroyed. Swelling thus occurred as a result of lithiation of the Si particles. It has been reported that the electrode thickness increases by approximately five to six times after full charging. ${ }^{8)}$ In Fig. 4(d), which illustrates the cell discharged from the state in Fig. 4(b) (SOC 40\%), the SEI and traces of Li intrusion are reduced, confirming Li desorption.

\subsection{TEM and STEM observations and EELS analysis after charging and discharging}

The thin film specimens of the surface layer and interior structure of the Si particles of the negative electrodes in the charged and discharged states observed via SEM were prepared using the FIB method, and TEM and STEM imaging as well as EELS analysis were carried out.

As shown in Fig. 5, a thin film specimen of the Si particles with $\mathrm{SOC}=40 \%$ was prepared and observed. Figure 5(a) shows the cross-sectional TEM image, and Fig. 5(b) shows an enlarged TEM image of the linear lithiated parts. The enlarged TEM image was acquired by irradiating along the [110] direction. Figure 5(b) also shows the electron beam diffraction (ED) patterns acquired from the areas labeled i) and ii). As shown by the arrows in Fig. 5(a), regularity can be observed in the morphology of the plate-like structures. This is due to the fact that lithiation accompanying charging occurs parallel to the $\langle 110\rangle$ direction due to the anisotropy of lithiation with respect to the crystallographic orientation of Si. From Fig. 5(b), it can be seen that the width of the plate-like structures ranges from 50 to $200 \mathrm{~nm}$. Comparing the nano-beam diffraction patterns obtained from the regions labeled i) and ii), marked by the white circles, a halo pattern can be observed from the region labeled ii), indicating that the plate-like structures have become amorphous due to lithiation. It is well known that lithiation generally causes amorphization of $\mathrm{Si}$ crystals, and, if the $\mathrm{Li}$ concentration 


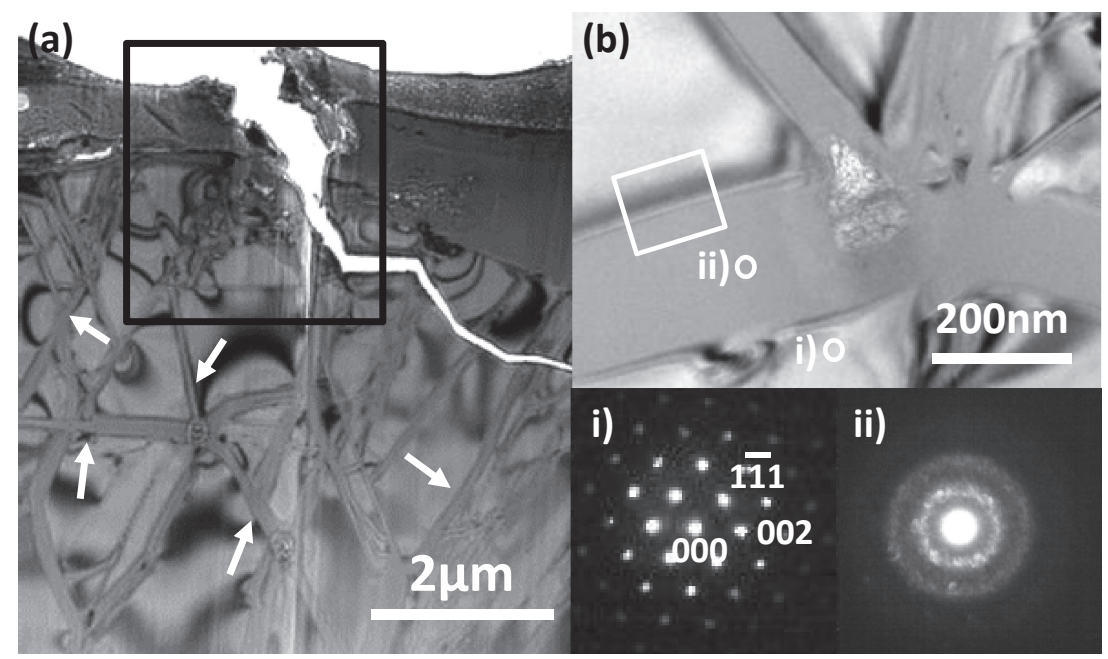

Fig. 5 (a) Cross-sectional TEM image of the Si negative electrode with SOC 40\%. The lithiated linear parts are indicated by arrows. (b) Enlarged image of lithiated linear parts observed in the [110] direction. The nano-beam diffraction patterns taken in the regions i) and ii) are also shown in figure.

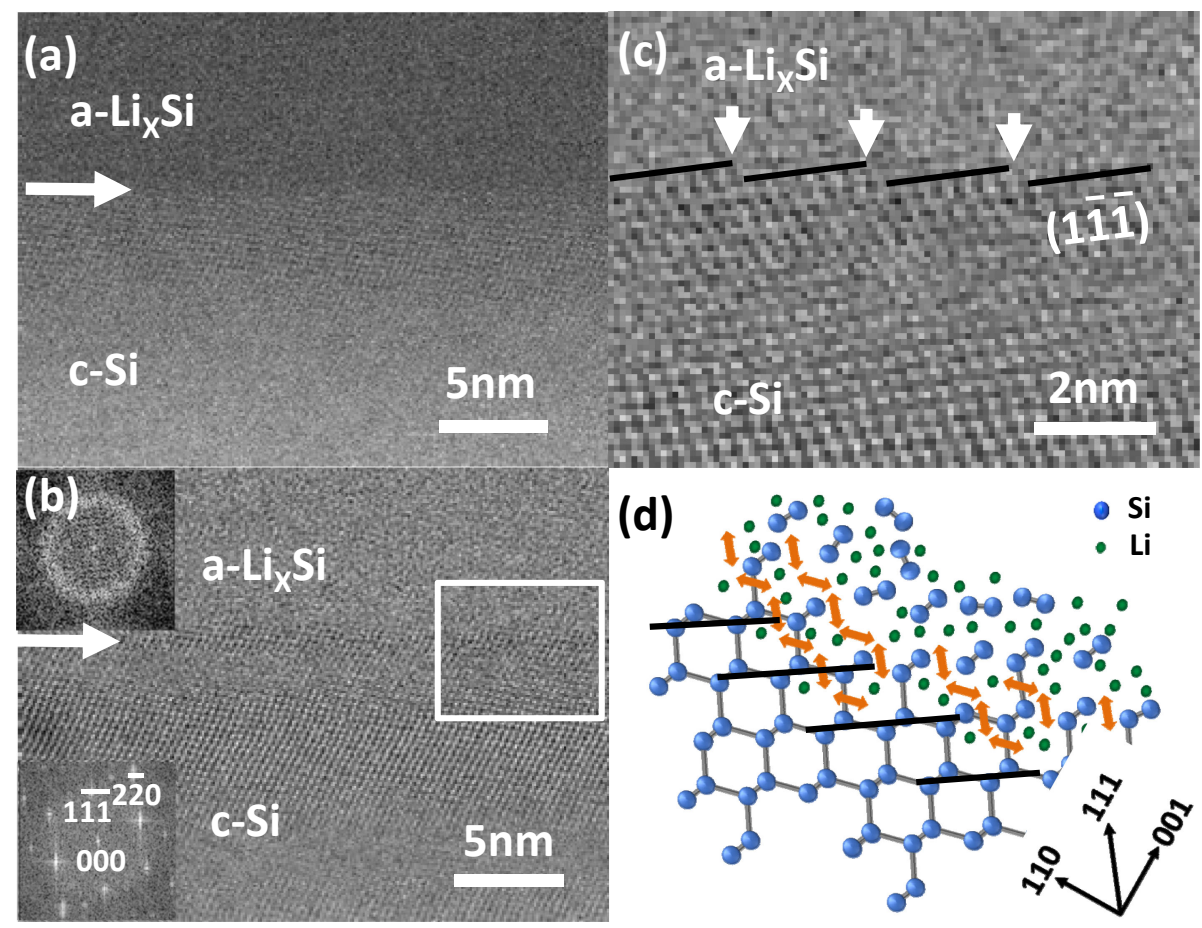

Fig. 6 (a) HAADF-STEM image of the region enclosed in a white square in Fig. 5(b). (b) BF-STEM image taken in the same area of Fig. 6(a). FFT images taken from upper-side $(\mathrm{a}-\mathrm{Li} \mathrm{S} \mathrm{Si})$ and lower-side (c-Si) are also shown in the figure. (c) High-resolution BF-STEM image of the region enclosed in a white square in Fig. 6(b). Ledge structure in one atomic layer at the interface between a-Li $\mathrm{Si}_{\mathrm{S}}$ and c-Si are indicated by the arrows. (d) Schematic image of the ledge structure formed by lithiated at a- $\mathrm{Li}_{\mathrm{x}} \mathrm{Si} / \mathrm{c}-\mathrm{Si}$ interface.

continues to increase after amorphization, a $\mathrm{Li}_{15} \mathrm{Si}_{4}$ phase ( $\mathrm{Cu}_{15} \mathrm{Si}_{4}$ prototype, Space group: I-43d) is formed. $\left.{ }^{5}\right)$

Figure 6(a) shows a high-angle annular dark-field scanning TEM (HAADF-STEM) image of the region enclosed by the white square in Fig. 5(b). Figure 6(b) is a bright-field scanning TEM (BF-STEM) image acquired in the same area as that in Fig. 6(a). Figure 6(c) is a high-resolution BFSTEM image of the region enclosed by the white square in Fig. 6(b), and Fig. 6(d) is a schematic image of amorphization by lithiation of Si. Since the image contrast in an HAADF-STEM image is proportional to the second power of the atomic number $z$, the contrast observed from the image in Fig. 5(a) originates from $\mathrm{Si}(z=14)$ for what concerns the white part. By contrast, the parts exhibiting black contrast, which consist of plate-like structures, are thought to include $\mathrm{Li}(z=3)$ in addition to $\mathrm{Si}$. Based on this, the region indicated by the arrows in the figure is believed to be the interface between a-Li $\mathrm{x}_{\mathrm{x}} \mathrm{Si}$ and c-Si. In Fig. 6(b), where the locations marked by the arrows indicate the interface between the lithiated and crystalline Si regions, a clear crystal lattice cannot be observed in the region with high Li concentration. Additionally, from the fast Fourier transform image of the lithiated and crystalline $\mathrm{Si}$ regions, the lithiated region displays a halo pattern indicative of amorphization. More- 


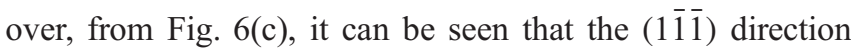
forms a step (ledge) structure in one atomic layer at the interface between $\mathrm{a}-\mathrm{Li}_{\mathrm{x}} \mathrm{Si}$ and $\mathrm{c}-\mathrm{Si}$, as indicated by the arrows.

Liu et al. investigated the formation of the a- $\mathrm{Li}_{\mathrm{x}} \mathrm{Si}$ phase by lithiation in Si nanowires via in situ TEM imaging of the lithiation process during the charging reaction. They reported that $\mathrm{Si}$ becomes amorphous due to lithiation, which causes separation (fracture) in one atomic layer through the movement of the ledge structure via a solid state reaction at the amorphous/crystalline interface. ${ }^{16)}$ In the present work, it is speculated that amorphization proceeds via movement of the interface through a solid state reaction originating from the edge of the $\{111\}$ facet (crystal wall) marked by the arrows in Fig. 6(c). Molecular dynamic calculations were used to investigate electrochemical lithiation, and the process whereby interfacial movement occurs due to delamination of the $\{111\}$ facets and subsequent amorphization when $\mathrm{Li}$ intrudes between adjoining $\{111\}$ plane layers was clarified at the atomic level. ${ }^{17)}$ Since the surface energy of the $\{111\}$ planes of $\mathrm{Si}$ is lower than that of other crystal planes, cleavage of the $\{111\}$ planes occurs more easily. Therefore, as shown in the schematic diagram in Fig. 6(d), it is thought that interfacial movement occurs through a process in which $\mathrm{Li}$ atoms occupy the tetrahedral sites of Si crystals. Thus, as the concentration of Li increases, amorphization proceeds via destruction of the Si crystal structure as the Si bonds between the $\{111\}$ planes are broken. This process appears as a zigzag pattern, as indicated by the arrows in Fig. 6(d). As the charge depth increases and the Li concentration in the Si crystals increases, an amorphous phase is formed through the destruction of the crystal lattice accompanying the increased lithiation at tetrahedral sites, and this region expands through preferential growth in the $\langle 110\rangle$ direction. $\left.{ }^{8}\right)$ The anisotropy of the linear regions with size of several microns shown in the SEM image in Fig. 4 and in the TEM image in Fig. 5 are likely to originate from this anisotropic growth of the amorphous phase. Amorphization caused by the solid state reaction originated during lithiation has also been confirmed by observation of the ledge structure in an ex-situ experiment using the negative electrode of an actual battery structure. This is consistent with the abovementioned in situ TEM imaging results for the lithiation of the Si nanowires obtained by Liu et al. Thus, the present work also demonstrates the effectiveness of experiments under non-atmospheric exposure conditions for the observation of the microstructure of $\mathrm{Si}$ negative electrodes.

Figure 7(a) shows the cross-sectional HAADF-STEM image of the near-surface area of the $\mathrm{Si}$ particles in the negative electrode with $\mathrm{SOC}=40 \%$, including the SEI, in the region enclosed by the black square in Fig. 5(a). Figure 7(b) displays the enlarged HAADF-STEM image of
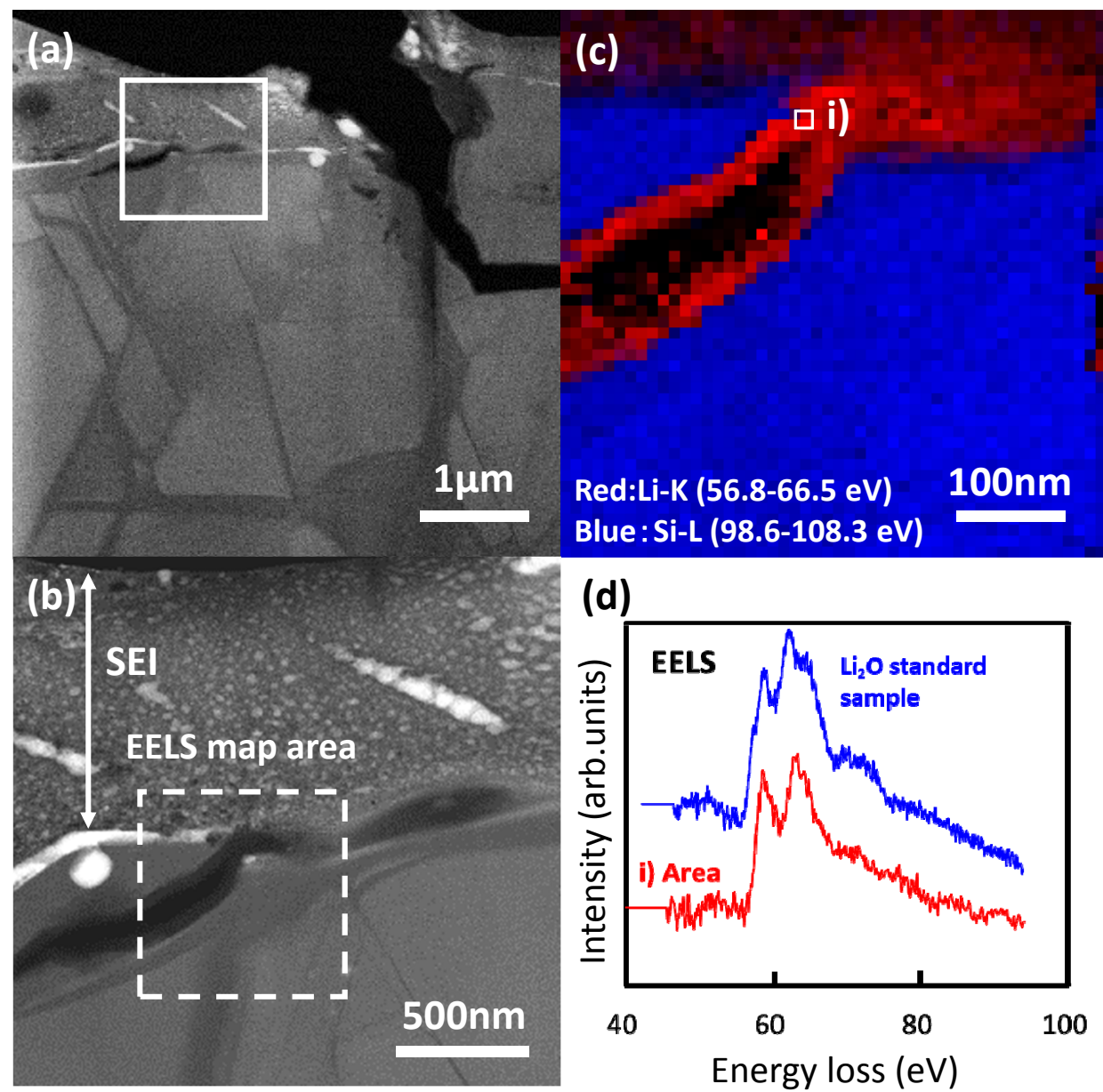

Fig. 7 (a) Cross-sectional HAADF-STEM image of the Si negative electrode with SOC 40\% enclosed in a black square in Fig. 5(a). (b) Enlarged image enclosed in a white square in Fig. 7(a). (c) Enlarged STEM-EELS map (red: Li, blue: Si) enclosed in a broken line in Fig. 7(b). (d) The EEL spectra obtained in an area i) in Fig. $7(\mathrm{c})$ and $\mathrm{Li}_{2} \mathrm{O}$ standard sample. 
the region enclosed by the white square in Fig. 7(a). Figure 7(c) illustrated the EELS map of the region enclosed by the white dashed line in Fig. 7(b), where red indicates $\mathrm{Li}$ and blue indicates Si. Figure 7(d) shows the EEL spectra obtained for the area labeled i) in Fig. 7(c). In Fig. 7(a), a black contrast is observed in the linear parts shown in Fig. 5(a), and the existence of a region with a high $\mathrm{Li}$ concentration can be confirmed. From the enlarged image in Fig. 7(b), it can be seen that a SEI with a thickness of approximately $1 \mu \mathrm{m}$ has formed, which is in excellent agreement with the results of the SEM observations in Fig. 4(b). Moreover, it is clear that the SEI also contains structures with size of several tens of nanometers and different compositions, which are indicated by the white contrast.

According to Peled et al., the SEI formed on the surface of a negative electrode as a consequence of the reaction between an electrolytic solution and $\mathrm{Li}$ is not a single oxide but is rather thought to be composed of inorganic compounds, such as $\mathrm{LiF}, \mathrm{Li}_{2} \mathrm{CO}_{3}$, and $\mathrm{Li}_{2} \mathrm{O}$, and organic compounds. ${ }^{13)}$ Therefore, an EELS area analysis (two-dimensional scan) was performed in the region enclosed by the dashed line, which includes the SEI that formed on the surface of the Si particles. Figure 7(c) indicates that the main component of the SEI is Li. Furthermore, in the spectra obtained for the area labeled i) in Fig. 7(c), a Li-K absorption edge was observed around $60 \mathrm{eV}$, and the absorption edge of the high energy side was larger than that of the low energy side. As this approximates the spectrum obtained from a $\mathrm{Li}_{2} \mathrm{O}$ standard sample, the main component of the SEI is considered to be $\mathrm{Li}_{2} \mathrm{O}$. However, the structure described above was not identified in detail, and $\mathrm{Ga}$ ions originating from the FIB process could also be present.

Subsequently, a thin film specimen consisting of a $\mathrm{Si}$ negative electrode discharged from $\mathrm{SOC}=40 \%$ was prepared via FIB in the same manner as in Fig. 7. Figure 8(a) shows the cross-sectional HAADF-STEM image of the nearsurface area of the Si negative electrode, including the SEI. Figure 8(b) presents the enlarged HAADF-STEM image of the region surrounded by the white square in Fig. 8(a). Figure 8(c) illustrates the EELS map of the regions surrounded by the dashed line in Fig. 8(b). Figure 8(d) shows the EEL spectra obtained for areas labeled i) and ii) in Fig. 8(c).

In Fig. 8(a), linear parts like those observed in the charged state in Fig. 7(a) are not observed, and it can be understood that $\mathrm{Li}$ has been removed from the interior of $\mathrm{Si}$ and diffused to the positive electrode during charging. Moreover, cracking has also occurred in the Si due to the volumetric contraction accompanying the discharge process. In the enlarged image
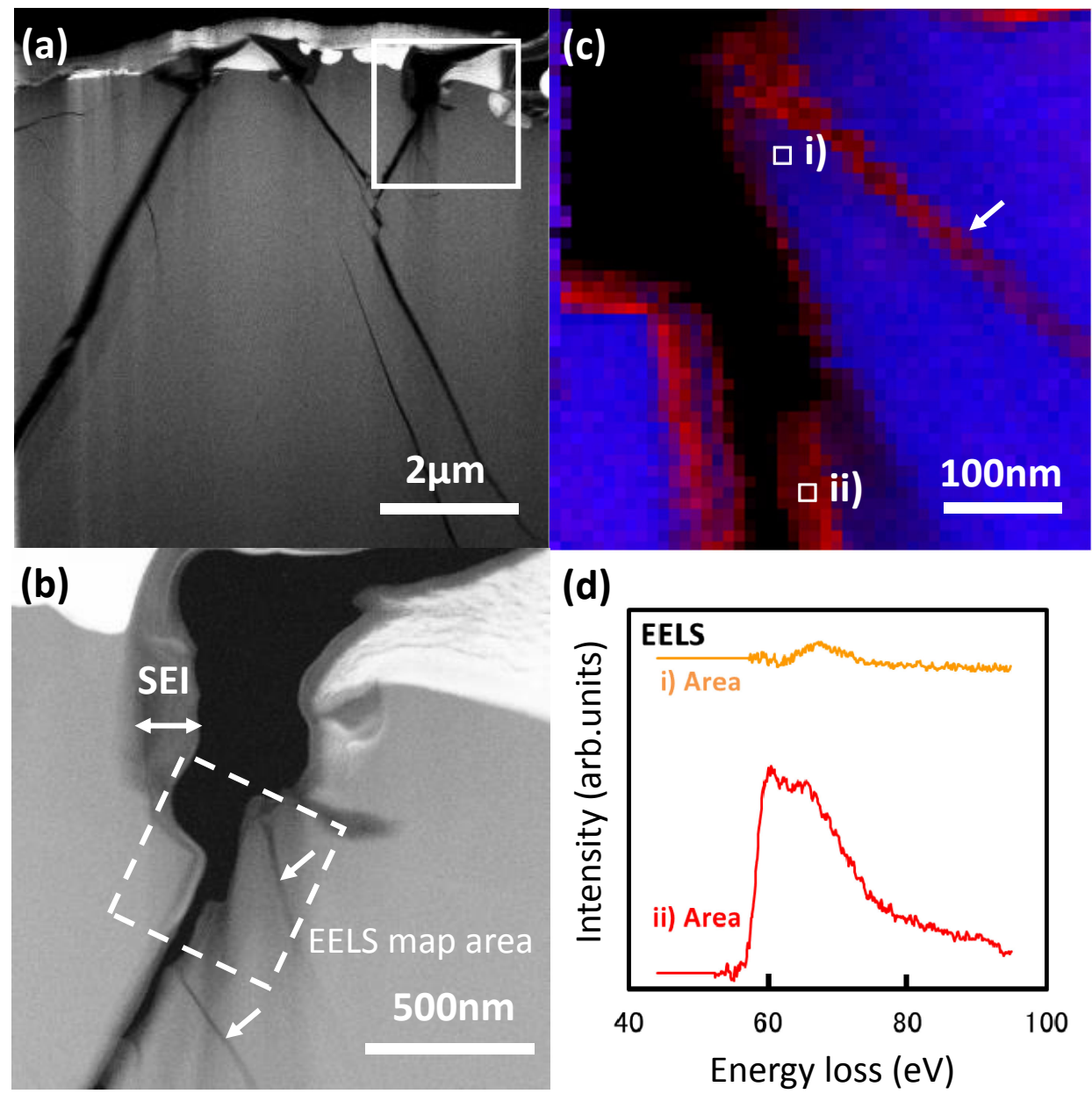

(d)

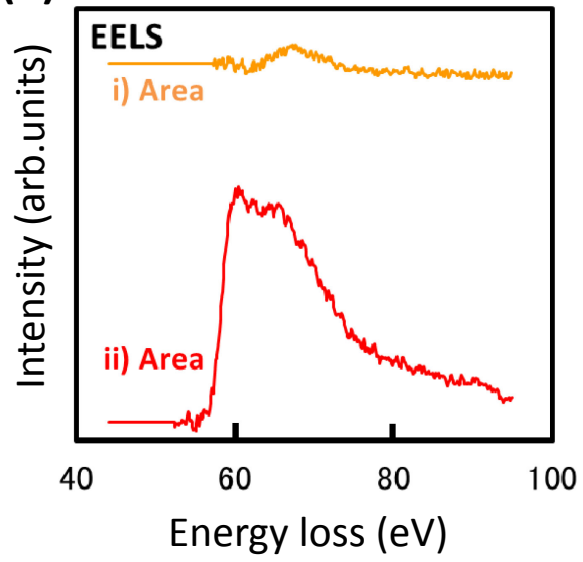

Fig. 8 (a) Cross-sectional HAADF-STEM image of the Si negative electrode discharged (SOC 40\% to 0\%) state. (b) Enlarged image enclosed in a white square in Fig. 8(a). Liner parts with gray contrasts are indicated by arrows. (c) Enlarged STEM-EELS map (red: Li, blue: Si) enclosed in a broken line in Fig. 8(b). Lithiated linear part is indicated by arrow. (d) The EEL spectra obtained in areas i) and ii) in Fig. 8(c). 
in Fig. 8(b), the thickness of the SEI is about $200 \mathrm{~nm}$ and is thus decreased after discharge. Black contrast can be observed at the $\mathrm{Si}$ negative electrode surface and at the positions indicated by the arrows, and differences in the composition have also occurred in Si after discharge. As a result of the EELS area analysis in the region enclosed by the dashed line, which includes the SEI, Fig. 8(c) shows that the SEI and the positions indicated by the arrows contain $\mathrm{Li}$. The EEL spectrum of the interior of the $\mathrm{Si}$ in the area labeled i) in Fig. 8(c) shows that the interior of the $\mathrm{Si}$ contains almost no Li; however, Li remains in the linear parts indicated by the arrows in the figure. The anisotropy of the $\mathrm{Li}$ diffusion path with respect to the crystal orientation of Si during charging (Li intrusion) has already been noted. However, since linear parts with decreased widths in comparison with the charged state remained after discharge, it is thought that also Li follows the same paths during discharge and diffuses toward the positive electrode via the SEI. The slight amount of Li observed in the EEL spectrum of the area labeled $\mathrm{i}$ ) is believed to originate from the $\mathrm{a}-\mathrm{Li}_{\mathrm{x}} \mathrm{Si}$ detected in the thickness direction of the TEM thin film, because the area around the interface between the Si region and the linear parts was investigated in the area analysis. Since the EEL spectrum obtained in the area labeled ii) approximates the spectrum shape of the $\mathrm{Li}_{2} \mathrm{O}$ standard sample observed in Fig. 7(d), it is thought that $\mathrm{Li}_{2} \mathrm{O}$ remained on the surface of the Si particles of the negative electrode, and the thickness of the SEI decreased after discharge.

During the charge/discharge reactions, Li ions are repeatedly inserted in and removed from the interior of the negative electrode via a process of diffusion through the SEI and its interface with the negative electrode. Although the SEI is formed after initial charging and then undergoes repeated adsorption/desorption as a result of charge/ discharge processes, it is known that the SEI also remains on the surface of the negative electrode after discharge, and its thickness gradually increases upon increasing the number of charge/discharge cycles. ${ }^{18)}$ As a result of the observation and comparison of the microstructure of the $\mathrm{Si}$ negative electrode during charge/discharging processes, a plate-like microstructure consisting of $\mathrm{a}-\mathrm{Li}_{\mathrm{x}} \mathrm{Si}$ formed via lithiation and a SEI with a thickness of approximately $1 \mu \mathrm{m}$ were observed in the interior of the $\mathrm{Si}$ crystal and on the surface layer, respectively. Since $\mathrm{Li}_{2} \mathrm{O}$ forms in close proximity to the $\mathrm{Si}$ negative electrode (i.e., immediately above the Si surface), it was assumed that $\mathrm{Li}_{2} \mathrm{O}$ is the primary component of the SEI. As confirmed via SEM and TEM observations, after discharge, the Li ions in $\mathrm{Si}$ are removed, the linear parts are extinguished, and the thickness of the SEI decreases to $1 / 5$ or less than that in the charged state. The SEI has the function of facilitating the reaction with the negative electrode; however, since it also causes irreversible loss of capacity, which affects the life of the battery, it is important to identify its component substances and clarify their formation sites and morphology. From Figs. 7(c) and 8(c), it can be seen that $\mathrm{Li}_{2} \mathrm{O}$ exists near the interface with the Si particles of the negative electrode. The properties of the SEI and its adhesion to the negative electrode are thought to depend strongly on its initial structure. ${ }^{14)}$ As a consequence, it was deemed appropriate to prepare TEM specimens via the FIB method in order to

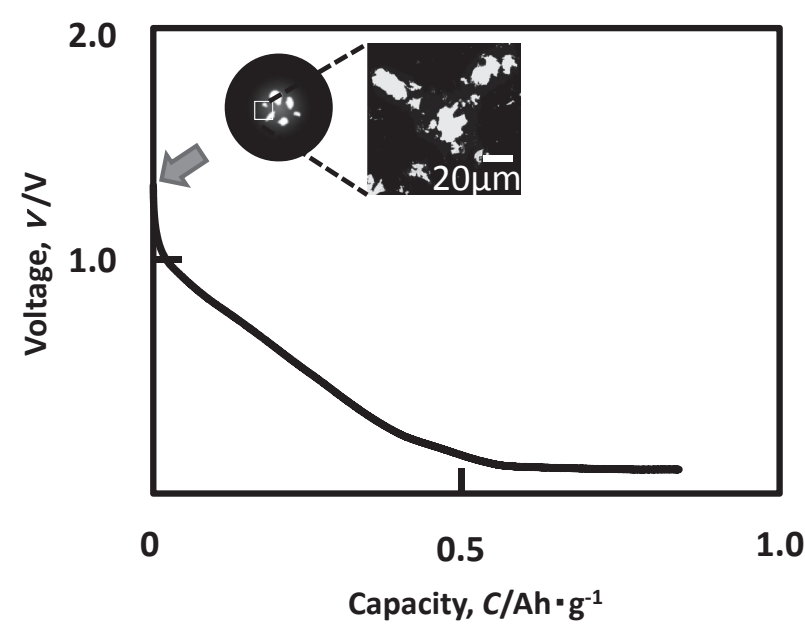

Fig. 9 Charging curve of TEM thin film (Si negative electrode) charged using the flat cell shown in Fig. 2. A schematic diagram of a TEM sample shape and a low-magnification TEM image of the thinned Si negative electrode are shown in the figure.

properly evaluate the observation position. In a previous experimental study on graphite negative electrodes, it was reported that the inner-SEI near the negative electrode interface has a high content of $\mathrm{Li}_{2} \mathrm{O}$ and other inorganic components, ${ }^{19)}$ and the fact that a similar tendency exists with different negative electrode active materials is very interesting.

\subsection{Charging of TEM thin films (Si negative electrode thin films) and TEM/STEM imaging and EELS analysis before/after charging}

When using FIB, the possibility of damaging the structure of the SEI is a concern because the thin film is prepared by high energy $(30 \mathrm{kV})$ irradiation of $\mathrm{Ga}$ ions on the sample material. Therefore, in the present study, TEM observations of the $\mathrm{Si}$ negative electrode before/after charging were conducted using the method described in section 2.5 in order to determine whether the SEI was damaged by FIB.

Figure 9 shows the charging curve of the TEM thin film prepared via FIB when charged with the flat cell shown in Fig. 2. The figure shows a schematic diagram of the shape of the TEM specimen and a low magnification TEM image of the observed specimen. Due to the possibility that the shape of the negative electrode might not be retained as a consequence of the swelling caused by charging and due to the fact that part of the specimen might be lost when using a thin film, the specimen was charged to $0.85 \mathrm{Ah} \cdot \mathrm{g}^{-1}$, equivalent to a SOC of approximately $20 \%$.

Figure 10 shows the TEM images of the SEI formed after charging to $\mathrm{SOC}=20 \%$ acquired for the same area before and after electron beam irradiation for $120 \mathrm{~s}$. When the region $(1.3 \mu \mathrm{m})$ enclosed by the white circle in the TEM image in Fig. 10(a) was irradiated, irradiation traces like those seen in Fig. 10(b) were observed. Since the shape of the SEI changed, changes in its structure were also considered possible. This indicates that the SEI is degraded via electron beam irradiation according to TEM observations. Basak et al. imaged $\mathrm{Li}_{2} \mathrm{O}_{2}$ powder particles via TEM under nonatmospheric exposure conditions and investigated in detail the reduction of $\mathrm{Li}_{2} \mathrm{O}_{2}$ to $\mathrm{Li}_{2} \mathrm{O}$ resulting from the increased 

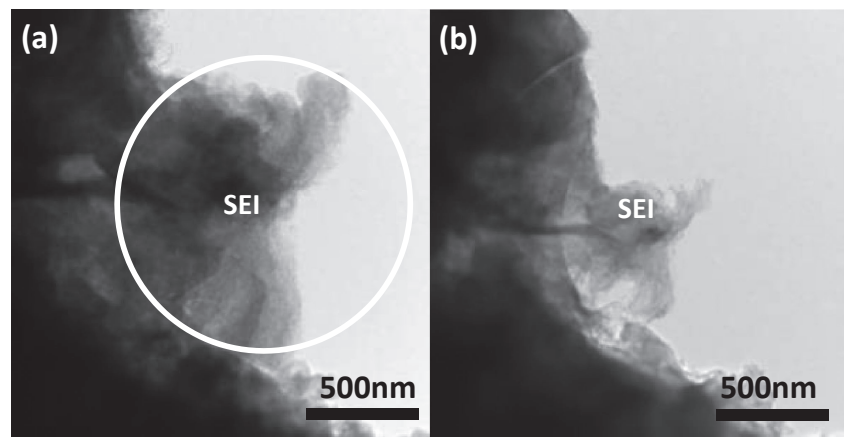

Fig. 10 TEM images of the SEI on the Si negative electrode with SOC $20 \%$ charged by the flat cell (a) before electron beam irradiation and (b) after electron beam irradiation for a $120 \mathrm{~s}$

electron beam irradiation time through EELS analysis. ${ }^{20)}$ The authors also reported that imaging at $200 \mathrm{kV}$ is more appropriate than at $80 \mathrm{kV}$ because the area of the inelastic scattering cross section is inversely proportional to the accelerating voltage. Accordingly, as shown in the experiment in Fig. 10, observations were conducted within a time of $60 \mathrm{~s}$ in order to avoid degradation of the SEI during imaging and ED when using this thin film specimen.

Figure 11(a) shows the TEM image before charging, whereas Fig. 11(b) illustrates the enlarged image corresponding to the area labeled i) in Fig. 11(a). Figure 11(c) displays the TEM image after charging to SOC $=20 \%$ for the same area as in Fig. 11(b), whereas Fig. 11(d) shows the enlarged TEM image after charging to SOC $=20 \%$ corresponding to the area labeled ii) in Fig. 11(a). Furthermore, the ED pattern and EEL spectrum obtained from the area labeled iii) in Fig. 11(c) are shown in Figs. 11(e) and 11(f), respectively. The observations and analysis were carried out within $60 \mathrm{~s}$ after electron beam irradiation, and the data were obtained after confirming that there was no degradation of the SEI. As shown in Fig. 11(a), oxides are not formed on the thin film specimen after ion milling. Additionally, from Fig. 11(b), it can be seen that handling the specimen under nonatmospheric exposure conditions is appropriate, as the $\mathrm{Si}$ surface layer is flat before charging. However, in comparison with the specimen surface condition before charging, surface irregularities can be seen in Fig. 11(c) after charging, and a SEI with a thickness reaching about $800 \mathrm{~nm}$ is locally formed. Moreover, as can be observed from Fig. 11(d), it is clear that SEI particles with a size of several tens of nanometers form on the surface of the Si negative electrode, and these particles can also be observed at other locations. In the ED pattern in Fig. 11(e), the (111), (220), (311), (400), and (331) Debye rings of $\mathrm{Li}_{2} \mathrm{O}$ (space group: $\mathrm{Fm} \overline{3} \mathrm{~m}$ ) can be observed. An investigation of the rings revealed that their spacings were $0.272,0.166,0.141,0.116$, and $0.106 \mathrm{~nm}$, respectively, which is consistent with existing knowledge concerning the lattice spacing of $\mathrm{Li}_{2} \mathrm{O}$. The results of the EELS analysis in Fig. 11(f) are also similar to the spectrum obtained from the standard $\mathrm{Li}_{2} \mathrm{O}$ sample and the spectrum obtained from the SEI in Fig. 7(d), supporting the conclusion that the SEI on the surface of the Si negative electrode after charging to $\mathrm{SOC}=20 \%$ is predominantly composed of $\mathrm{Li}_{2} \mathrm{O}$.

These results clarify the fact that $\mathrm{Li}_{2} \mathrm{O}$ forms in close proximity to the surface of the Si negative electrode during the reaction, regardless of the charging method, and the SEI formed during the charging reaction consists mainly of $\mathrm{Li}_{2} \mathrm{O}$. Based also on this fact, the TEM specimen preparation conditions using FIB ion milling for the sample with SOC $=40 \%$ shown in Figs. 7 and 8 can be considered to be appropriate for SEI imaging. Basak et al. pointed out that STEM observations, which enable lower irradiation current, are suitable for $\mathrm{Li}_{2} \mathrm{O}_{2}$ imaging. ${ }^{20}$ ) Likewise, the results of the present study show that $\mathrm{Li}_{2} \mathrm{O}$ is degraded under TEM observations, suggesting that STEM-EELS is an appropriate technique for observation of Si negative electrodes charged to $\mathrm{SOC}=40 \%$ and discharged.
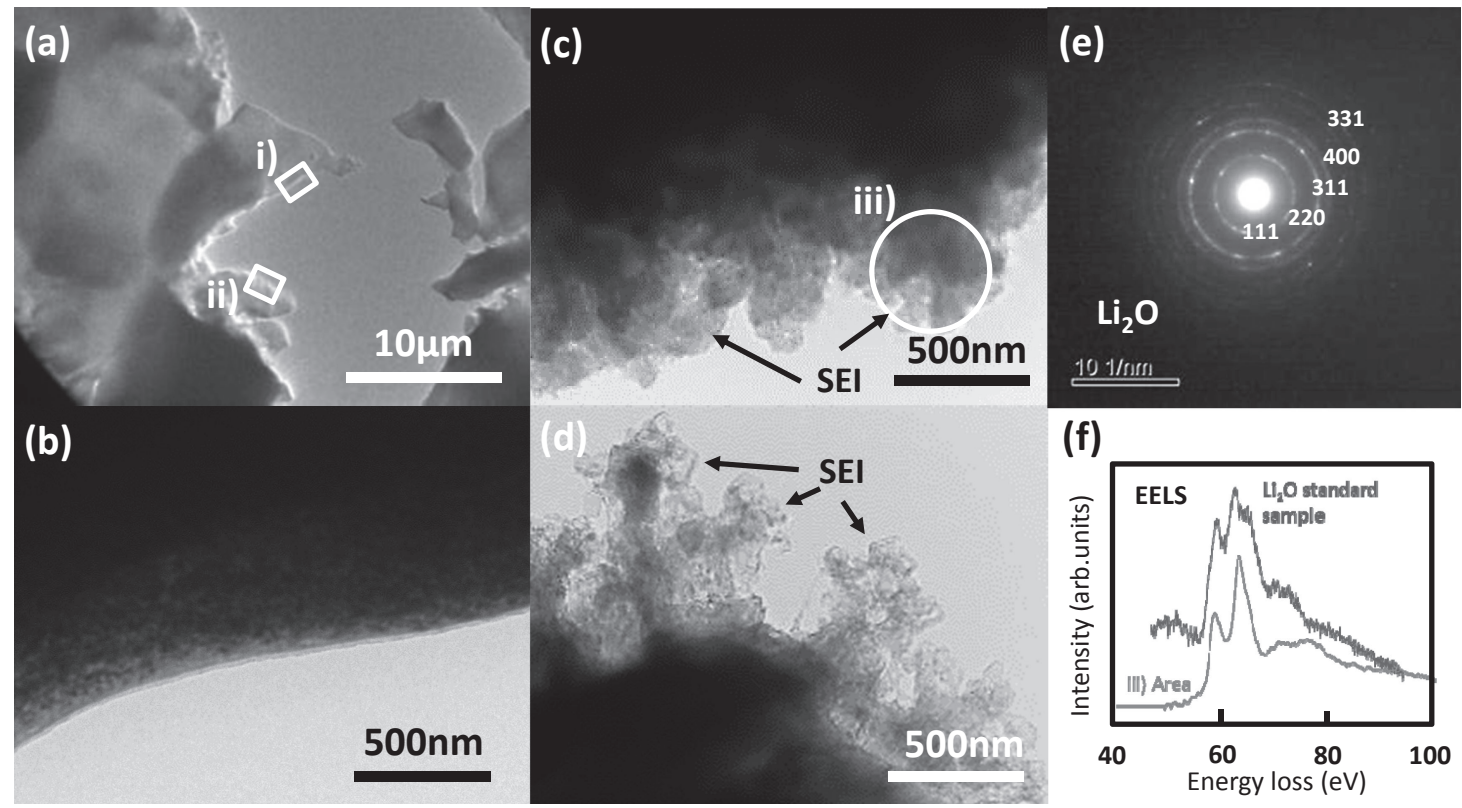

Fig. 11 TEM images of (a) before charge, (b) enlarge image of i) in a white square in Fig. 11(a). (c) TEM image of SOC 20\% taken from same area of Fig. 11(b). (d) TEM image of SOC 20\% taken from same area of ii) in Fig. 11(a). (e) ED pattern and (f) EELS spectrum obtained in area iii) in Fig. 11(c). The EEL spectrum of the $\mathrm{Li}_{2} \mathrm{O}$ standard sample also shown in the Fig. 11(f). 


\section{Conclusions}

(1) By comparing the differences in the Si particles in the Si negative electrodes after charging/discharging via SEM imaging, linear parts accompanying lithiation and a SEI surrounding them were observed in $\mathrm{Si}$ after charging. After full charging to $\mathrm{SOC}=100 \%$, the $\mathrm{Si}$ particles were unable to maintain their shape before charging and were destroyed by the growth of the SEI and the swelling caused by the intrusion of $\mathrm{Li}$ into $\mathrm{Si}$. After discharge, the linear parts and SEI decreased, and the desorption of Li from the Si interior was confirmed.

(2) BF- and HAADF-STEM imaging of the linear parts observed in the interior of the $\mathrm{Si}$ negative electrode with $\mathrm{SOC}=40 \%$ revealed that these parts have an amorphous phase consisting of $\mathrm{Li}$ and $\mathrm{Si}$. Additionally, these investigations confirmed the existence of a ledge structure causing interfacial movement as amorphization proceeds following the destruction of the crystal structure as the Si bonds between the $\{111\}$ planes are broken in the solid state reaction at the amorphous/ crystalline interface.

(3) The STEM-EELS observations of the specimen charged to SOC $=40 \%$ with an actual battery showed that $\mathrm{Li}_{2} \mathrm{O}$ formed near the $\mathrm{Si}$ negative electrode interface (immediately above the $\mathrm{Si}$ ), indicating that $\mathrm{Li}_{2} \mathrm{O}$ is the main component of the SEI. A SEI with a thickness of about $1 \mu \mathrm{m}$ was observed on the surface of the Si particles of the negative electrode, and the thickness of this SEI decreased to $200 \mathrm{~nm}$ or less after discharge. Linear parts containing $\mathrm{Li}$ were also observed in $\mathrm{Si}$ after discharge; however, since their width was decreased in comparison with that in the charged state, it is speculated that Li ions also follow the charging path (Li insertion paths) during discharge and diffuse toward the positive electrode via the SEI.

(4) Microstructural observations of a TEM thin film which was directly charged to $\mathrm{SOC}=20 \%$ in a flat cell showed that the SEI was degraded by electron beam irradiation (120s) during TEM imaging. By performing TEM observations with limited duration, a SEI with a thickness of approximately $800 \mathrm{~nm}$ was observed on the surface of the Si negative electrode. Electron diffraction and EELS analysis revealed that this SEI consisted mainly of $\mathrm{Li}_{2} \mathrm{O}$.
(5) STEM-EELS observations of $\mathrm{Si}$ negative electrodes charged/discharged with the actual battery and TEM observations of a Si negative electrode directly charged on a TEM thin film showed that $\mathrm{Li}_{2} \mathrm{O}$ forms near the interface with the $\mathrm{Si}$ negative electrode (immediately above the Si), and the SEI formed on the Si negative electrode during the charging reaction consisted predominantly of $\mathrm{Li}_{2} \mathrm{O}$. Since the SEI is degraded by electron beam irradiation, STEM observation using irradiation with a lower irradiation current is considered appropriate.

\section{REFERENCES}

1) R. Van Noorden: Nature 507 (2014) 26-28.

2) U. Kasavajjula, C. Wang and A.J. Appleby: J. Power Sources 163 (2007) 1003-1039.

3) W. Wan, Q. Zhang, Y. Cui and E. Wang: J. Phys. Condens. Matter 22 (2010) 415501.

4) K. Zhao, W.L. Wang, J. Gregoire, M. Pharr, Z. Sou, J.J. Vlassak and E. Kaxiras: Nano Lett. 11 (2011) 2962-2967.

5) M.K.Y. Chan, C. Wolverton and J.P. Greeley: J. Am. Chem. Soc. 134 (2012) 14362-14374.

6) E.D. Cubuk, W.L. Wang, K. Zhao, J.J. Vlassak, Z. Suo and E. Kaxiras: Nano Lett. 13 (2013) 2011-2015.

7) S.W. Lee, M.T. McDowell, J.W. Choi and Y. Cui: Nano Lett. 11 (2011) 3034-3039.

8) Y. Shimauchi, S. Ikemoto, S. Ohmori and T. Itoi: Mater. Trans. 60 (2019) 2328-2335.

9) D. Aurbach: J. Power Sources 89 (2000) 206-218.

10) P. Verma, P. Maire and P. Novak: Electrochim. Acta 55 (2010) 63326341.

11) Y. Wang, S. Nakamura, M. Ue and P.B. Balbuena: J. Am. Chem. Soc. 123 (2001) 11708-11718.

12) Y. Wang, S. Nakamura, K. Tasaki and P.B. Balbuena: J. Am. Chem. Soc. 124 (2002) 4408-4421.

13) E. Peled, D. Golodnisky and G. Ardel: J. Electrochem. Soc. 144 (1997) L208-L210.

14) K. Ushirogata, K. Sodeyama, Z. Futera, Y. Takeyama and Y. Okuno: J. Electrochem. Soc. 162 (2015) A2670-A2678.

15) M. Nie, D. Chalasani, D.P. Abraham, Y. Chen, A. Bose and B.L. Lucht: J. Phys. Chem. C 117 (2013) 1257-1267.

16) X.H. Liu et al.: Nano Lett. 11 (2011) 3312-3318.

17) Y.Q. Tong, K. Gutjahr, S. Hopfe, U. Gosele and T.H. Lee: Appl. Phys. Lett. 70 (1997) 1390-1392.

18) Y. Tao, S. Shigeki, T. Ozaki, T. Inamura, H. Yoshida and R. Okuyama: GS Yuasa Technical Report, 10(2) (2013) 8-15.

19) P. Niehoff, S. Passerini and M. Winter: Langmuir 29 (2013) 5806 5816.

20) S. Basak, J. Jansen, Y. Kabiri and H.W. Zandbergen: Ultramicroscopy 188 (2018) 52-58. 